\title{
El "misterio incomprensible" de la vida: Lugar y estatuto de la figura de lo orgánico para la comprensión de la razón y del Absoluto en la filosofía de Hegel
}

\author{
SANDRA VIVIANA PALERMO \\ Consejo Nacional de Investigaciones Científicas y Técnicas \\ Universidad Nacional de Río Cuarto
}

DOI: $10.36446 /$ rlf2021176

\begin{abstract}
Resumen: El objetivo del presente trabajo es analizar y determinar el estatuto y el lugar de la vida para la concepción hegeliana de la razón, como así también la relación entre tales caracteres y el despliegue sistemático. La hipótesis que se pone en juego es que el desdoblamiento del concepto de vida en la Lógica -en la cual la vida aparece como inmediata existencia de la idea que ha de ser superada en el conocer y en la idea absoluta, reapareciendo al mismo tiempo en la idea absoluta como lo que la define en su más íntimo carácter y en su especificidad-anticipa y recoge el lugar de la vida dentro del cuadro sistemático. En el sistema hegeliano, de hecho, la vida se presenta como momento constitutivo y genético mas no definitorio del espíritu, pues ha de ser superado por aquel y, al mismo tiempo, como la verdadera y más propia especificidad del espíritu.
\end{abstract}


Palabras clave: unidad orgánica, proceso, actividad, contradicción.

\title{
The "Incomprehensible Mystery" of Life: Place and Statute of the Figure of Organic for the Understanding of Reason and Absolute in Hegel's Philosophy
}

\begin{abstract}
The aim of this work is to examine and determine the status and place of life for Hegel's concept of reason, as well as the relationship between such determinations and the systematic structure. The doubling of the concept of life in Logic, in which life appears as the immediate existence of the idea that has to be overcome in the idea of cognition and in the absolute idea and, at the same time, reappears in the latter, defining its very nature and specificity, anticipates and collects the place of life within the systematic framework; here it appears as a constitutive and genetic but not defining moment of the spirit, since it has to be overcome by it and, at the same time, as the true and most proper peculiarity of the spirit.
\end{abstract}

Key-words: organic unity, process, activity, contradiction.

1.

T as imágenes orgánicas atraviesan la obra de Hegel desde sus pri-

meros esbozos $^{1}$-en los que la vida asume el carácter cósmico-metafisico de unificación de opuestos (cfr. GW 2: 341-348; EJ: 399-405)- hasta los textos de la madurez -en los que lo verdaderamente viviente no solo es el espíritu en su punto más alto (cfr. Enc. \$379Z; GW 12: 236) sino también la verdad. En tanto que proceso de realización de sí en lo otro de sí, la verdad es vida y es lo viviente; de ahí que, en el Prefacio a la Fenomenología del espíritu,

${ }^{1}$ Los textos de Hegel se citan de los Gesammelte Werke (1968 y ss.), in Verbindung mit der Hegel-Kommission der Rheinisch-Westfälischen Akademie der Wissenschaften und dem Hegel-Archiv der Ruhr-Universität Bochum-Düsseldorf (Hamburg: Meiner). Los mismos serán citados como $G W$, seguido por el número del volumen y el número de página. A tales referencias seguirá la referencia de la traducción al español, allí donde la hubiere. Las siglas correspondientes a las traducciones al español se consignan en la bibliografía al final del texto. En el caso de la Enciclopedia de las ciencias filosóficas, se hará referencia al número del apartado. 
Hegel ilustre el "desarrollo progresivo" de la misma a través de la diversidad de los distintos sistemas filosóficos, recurriendo al desenvolvimiento de la planta, que despliega y realiza lo que ella es en germen. Así como cada instancia del proceso de crecimiento de la planta comporta el desplazamiento y la negación de la instancia precedente, pero al mismo tiempo la implica y todas ellas, en su conjunto, constituyen los órganos de una única individualidad viviente, también la verdad se desplegaría, según el filósofo, en una serie de momentos contrapuestos y diferentes que constituyen su misma realización ( $f f r$. GW 09: 10; FdE: 57). Justamente porque implica exposición, proceso, intercambio y asimilación de lo otro de sí, movimiento de realización y actividad conforme a fin, la verdad se muestra como "sustancia viviente".

En los últimos años muchos estudiosos han insistido sobre la importancia del modelo orgánico para la estructuración del idealismo hegeliano y para su concepción del concepto y del Absoluto. Dina Edmundts y RolfPeter Horstmann, por ejemplo, sostienen que la tarea de la filosofia, para Hegel, es el autoconocimiento de la razón y que el filósofo de Stuttgart piensa tal proceso en conexión con el modelo orgánico de desarrollo: "la representación fundamental que guía su [la de Hegel] concepción es que se debe concebir la razón según el modelo del organismo viviente" (Edmundts y Horstmann 2002: 11). También Thomas S. Hoffmann, en su Hegel: Una propedéutica, insiste en la importancia de la vida para la comprensión de la "ontología dialéctica" hegeliana. La obra del filósofo se desplegaría entonces a partir de la noción de vida que él desarrolla en los escritos juveniles, en los que lo viviente no es "'ser' en el sentido de algo que está ahí positivamente, sino ser en el sentido de relación" (Hoffmann 2014: 106); una relación que no puede ser comprendida bajo la lógica intelectiva de la subsunción y que justamente por eso impone un nuevo modo de pensar el vínculo entre universal y particular, a partir del cual lo universal es tal solo como individualizado: la vida es en el viviente y, al mismo tiempo, el viviente individual se constituye en cuanto es él mismo la vida que es (cfr. GW 02: 345-346; EJ: 399-400).

También Frederick Beiser reconoce en el concepto de organismo una figura clave para comprender el idealismo hegeliano que ha de ser definido, según el autor, como un "monismo vitalista" (Beiser 2003: 141). Tanto en su Hegel como en otros trabajos dedicados a la filosofia de la naturaleza hegeliana, Beiser sostiene que la visión orgánica del mundo, es decir la consideración del mundo como un "inmenso y singular organismo viviente", es el fundamento del idealismo objetivo, la adquisición teórica a partir de la cual el filósofo de Stuttgart se propondría superar el dualismo kantiano-fichteano de sujeto-objeto y naturaleza-espíritu, en la medida en que tales opuestos pueden ahora ser pensados como "grados diferentes de organización y de- 
sarrollo de una única fuerza viviente" (Beiser 2005: 105). Beiser insiste en que si bien el concepto de "organismo" es "prima facie una mera metáfora", adquiere sin embargo un significado "más preciso y técnico" en la obra de Hegel (Beiser 2005: 95)²; significado que se articularía sobre la base de las disquisiciones kantianas acerca del Naturzweck y de la noción de "universal sintético", esta última introducida por el pensador de Königsberg en el $\$ 77$ de la Kritik der Urteilskraft con el objeto de dar cuenta de una estructura en la cual el todo contiene el "fundamento de la posibilidad de la forma del mismo y del enlace de las partes perteneciente a tal todo", es decir, con el objeto de expresar la exigencia de una unidad capaz de enlazar en sí y consigo partes que de otro modo resultarían extrínsecas y contingentes respecto de aquella ( $A$ A 05: 404-410; CJ:268-275). De nuevo, como para Hoffmann, el concepto de organismo, leído a la luz del concepto kantiano de Naturzweck, habilita un nuevo modo de pensar la relación universal-particular.

Parece moverse en una dirección similar Karen Ng, que en su reciente texto Hegel's Concept of Life, afirma que en Hegel la imagen del organismo no ha de ser entendida simplemente como una metáfora -la razón "no es como la vida, sino que es actividad dinámica y viviente en constante desarrollo" (Ng 2020: 3)-, y que la vida opera como "centro orientador" de la filosofia de Hegel permitiéndole articular un concepto nuevo de idealismo (Ng 2020: 3). Por último, dentro de esta contextualización general acerca del lugar de la figura del organismo en Hegel, no se puede dejar de citar el trabajo de Annette Sell, Der lebendige Begriff: Leben und Logik bei G.W.F. Hegel. Aun restringiendo su campo de análisis a la idea lógica de la vida, este texto ofrece algunos puntos de apoyo interesantes, en la medida en que presenta la "vida natural como modelo para el movimiento del concepto y con ello para la dialéctica" (Sell 2013: 19), insistiendo, sin embargo, en que esto no implica ninguna naturalización del concepto o del pensar en $\mathrm{Hegel}^{3}$. La noción de vida posee, según la autora, un rol sistemático decisivo, como así también una función metodológica importante: "la vida, en sus diferentes formas, también en sus formas naturales, representa un pensamiento constitutivo para la lógica dialéctica" (Sell 2013: 196). Tampoco para Sell se trata entonces de un concepto simplemente metafórico, es decir, una imagen utilizada en lugar de otra cosa, pues en ese caso no tendría espacio en la lógica hegeliana; una lógica que pretende caracterizarse justamente mediante el

\footnotetext{
2 Sobre el organismo animal como "modelo analógico" para la estructuración del sistema hegeliano, cfr. Bodei 2014: 338-339.

${ }^{3}$ Habla en cambio de "naturalización" del concepto en Hegel Songsuk Susan Hahn, para la cual la estructura orgánica es la base sobre la cual Hegel piensa el concepto (Hahn 2007: 11).
} 
"concepto viviente" y no como exposición de formas conceptuales vacías, rígidas y muertas".

Aun reconociendo la necesidad para una lógica como la hegeliana de contener una exposición del concepto de vida y, justamente en virtud de la convicción de que Hegel orienta su concepción de las determinaciones del pensar a partir del modelo de la vida natural como "eigentliches Leben", Sell sostiene que tal orientación "se refiere al movimiento dialéctico del concepto que es en última instancia un concepto de la subjetividad" (Sell 2013: 18). También Beiser termina por aseverar la insuficiencia del concepto de organismo para dar cuenta de la filosofia hegeliana: "Para Hegel, el espíritu no es solo vida sino algo más: autoconciencia de la vida" (Beiser 2005: 110). En este sentido, para el autor, organismo es concepto fundamental pero no suficiente para dar cuenta de la especificidad de la filosofia hegeliana que no puede ser identificada con la filosofia de Schelling, teniendo su punto de confluencia no en la naturaleza sino en la filosofia, donde "espíritu" adquiere un significado fundamentalmente cultural e histórico.

Dentro de este complejo entramado interpretativo, el objetivo del presente trabajo es analizar y determinar el estatuto y el lugar de la vida para la concepción hegeliana de la razón, como así también la relación entre tales caracteres y el despliegue sistemático. La tesis que intentamos sostener es que la concepción hegeliana de la vida a partir de la estructura del Zweck como concepto especulativo que impone una nueva relación entre causa y efecto, universal y particular, todo y partes, permite un desdoblamiento de tal concepto; tal desdoblamiento, a nuestro modo de ver, halla expresión en la Lógica, en la cual la vida aparece como primera y más inmediata existencia de la idea que ha de ser superada en el conocer y en la idea absoluta y, al mismo tiempo, reaparece en el capítulo sobre la idea absoluta, definiéndola en su más íntimo carácter y en su especificidad. En segundo lugar, el desdoblamiento del concepto de vida en la lógica anticipa y recoge el lugar de la vida dentro del cuadro sistemático, donde la misma se presenta como momento constitutivo y genético mas no definitorio del espíritu, pues ha de ser superado por aquel y, al mismo tiempo, como la verdadera y más propia especificidad del espíritu.

A partir de los objetivos trazados, el texto se articula en cuatro partes: en primer lugar, se intenta poner brevemente en evidencia la existencia de

\footnotetext{
${ }^{4}$ A este cuadro general debe sumarse el texto de Stefania Achella (2020) al cual tuvimos acceso solo al final de la redacción del presente texto. Tampoco faltan voces que nieguen la vocación "organicista" de la filosofía hegeliana (cfr. Pippin 1989: 4, 61-62 y 66; y Kreines 2013: 111-154).
} 
un doble movimiento razón-vida en la filosofia kantiana del cual la filosofía de Hegel se presentaría como una radicalización $(\$ 2)$; en segundo lugar, el trabajo analiza el lugar y estatuto de la vida en la Ciencia de la lógica, mostrando el desdoblamiento de la vida en tal esfera (\$3); en tercer lugar, se intenta dar cuenta de las implicancias, en el nivel sistemático, de la doble ubicación de la vida en la esfera lógica, poniendo atención en el significado de tal desdoblamiento para la relación espíritu-naturaleza (\$4); finalmente, el trabajo retoma la cuestión del doble movimiento razón-vida interrogándose acerca del significado de las adquisiciones precedentes para el concepto hegeliano de razón (\$5). La doble ubicación de la vida parecería indicar una doble estructura de la razón en Hegel, en virtud de la cual la misma ha de ser pensada en los términos de lo orgánico y, al mismo tiempo, no puede agotarse en aquel modelo. En este sentido la razón no se presenta solo como Zweck o Selbstzweck sino que se comporta como actividad que pone y establece fines. Esta doble caracterización resulta fundamental para comprender la especificidad del concepto hegeliano de Denken, como fuerza que anima y estructura el mundo, mas que se realiza como tal solo en la subjetividad pensante.

\section{2.}

— n los parágrafos 64 y 65 de la Crítica de la facultad de juzgar, Kant que un objeto sea considerado fin de la naturaleza. Tales condiciones, según el filósofo de Königsberg, responden a una reciprocidad de causa-efecto o parte-todo a la que el entendimiento no tiene acceso cognoscitivo, pues no la puede comprender sin contradicción. Para que algo pueda ser subsumido bajo el concepto de Naturzweck, escribe Kant, es necesario, en primer lugar, que "las partes (por su existencia y por la forma) solo sean posibles por su referencia al todo" ( $A A$ 05:373; CJ: 233); en segundo lugar, que "las partes se unan para la unidad de un todo de suerte que entre sí sean alternativamente causa y efecto de su forma". Esto significa que se necesita -aclara Kant- que todas las partes, tanto respecto de su forma como de su enlace o conexión, se produzcan entre sí, "formando así por causalidad propia un todo cuyo concepto pueda juzgarse, a su vez, inversamente, causa de ese cuerpo según un principio" ( $A A$ 05: 373; CJ: 233). Dicho de otro modo, el concepto de fin, más específicamente, de fin natural, requiere una reciprocidad causal todo-parte que en su más estricta inmanencia excluye que la cosa pueda ser entendida como "producto de una causa racional distinta de la materia (de las partes) del producto mismo; causa cuya causalidad (en la producción y enlace de las partes) se determina por su idea de un todo así posible" ( $A A 05$ : 
373; CJ: 233). En este último caso estaríamos ante un producto del arte y no ante un producto natural que se pueda definir también fin de la naturaleza.

Según la Analítica de la facultad teleológica de juzgar, entonces, aquel objeto que haya de ser subsumido bajo el concepto de fin natural deberá presentarse no solo como organizado, sino además como autoorganizado $(A A$ 05: 374; CJ: 234). A lo largo de la Analítica y de la Dialéctica de la facultad teleológica de juzgar Kant insiste acerca de la estructura lógica propia del concepto de Naturzweck, presentando la misma mediante la figura de la causa y efecto de sí (cfr. $A A$ 05: 370; $C J: 230$ ), mas también como relacionalidad recíproca de todo y partes ( $f r$. $A A$ 05:373; CJ: 233) y vinculando estas formas no intelectivas de relación al enlace de un universal que unifica consigo lo particular también en aquello que este último presenta como contingente y por lo tanto imposible de determinar a priori desde lo universal (cfr. AA 05: 404; CJ: 268269). Sin pretender reducir la estrategia kantiana a una única línea argumentativa, se puede resumir la posición del filósofo de Königsberg afirmando que, para él, el concepto de fin natural resulta ajeno al entendimiento y sus leyes constitutivas, pues para acceder a él necesitamos recurrir a un "principio de la razón" (Vernunftprinzip) puesto a disposición de la facultad de juzgar, que sirve como principio regulativo y no constitutivo o determinante para poder reflexionar sobre ciertos objetos dados en la experiencia.

En este sentido, el concepto de Zweck es un concepto propio de la razón (cfr. $A A$ 05:372; $C J: 232$ ) que sin embargo nosotros estamos autorizados a aplicar a ciertos objetos dados en la experiencia -más específicamente, a los seres organizados de la naturaleza- de forma solamente regulativa o reflexionante; es decir, mediante este predicado nosotros simplemente decimos que la peculiaridad de nuestras facultades cognoscitivas nos impone juzgarlos como articulados según fines, sin pretender sostener que los mismos son efectivamente (= objetivamente) fines de la naturaleza (cfr. AA 05: 397-398; CJ: 262-263). En otras palabras, recurrimos a un concepto habilitado por nuestra razón que la facultad de juzgar moviliza para darse una regla a sí misma en la investigación de ciertas leyes y formas empíricas de la naturaleza, y que por lo tanto no pretende ninguna validez objetiva.

Ahora bien, valdría la pena notar que este movimiento conceptual resulta inverso al de la Crítica de la razón pura, pues mientras que en la primera Crítica Kant se servía de la imagen del organismo para dar cuenta de la estructura de la razón (cfr. KrVA 834, 845; B XXIII, 862, 863; CRP 26, 846, $847)^{5}$, en la Crítica de la facultad de juzgar el filósofo recurre a la razón, por

\footnotetext{
${ }^{5}$ Véase también $A A$ 03: 264; Prol. 35.
} 
lo menos a un concepto constitutivo de la misma -el de fin- para poder explicar a los seres organizados de la naturaleza. Este doble movimiento kantiano - de la razón al organismo y del organismo a la razón- resulta fundamental para encuadrar el enfoque que anima la concepción hegeliana. En primer lugar, Hegel no solo se servirá del concepto de organismo como relacionalidad recíproca de parte y todo, sino que además instaurará, sobre el concepto kantiano de "universal sintético" como totum -en el que el todo no es resultado de la suma de partes que tienen una consistencia por fuera del todo, sino fundamento de la forma y del enlace de las partes- su concepto de "universal concreto" (cfr. Beiser 2005: 96; Palermo 2017: 245-265). En segundo lugar, la reflexión kantiana acerca de la estructura del Zweck como concepto constitutivo de la razón y su íntima relación con el organismo viviente se presentará, para Hegel, como el perno sobre el cual hacer girar una nueva concepción de la razón y del pensar que permita, con Kant, ir más allá de Kant (cfr. Chiereghin 1990:127-229) consintiéndole así superar las rígidas columnas de Hércules del criticismo.

Lo que intentamos decir es que también en Hegel encontramos un movimiento relacional de doble dirección entre razón y vida (u organismo) la diferencia entre ambos autores reside en que mientras que en Kant tal movimiento parece presentarse como "interrumpido", pues allí donde se utiliza al organismo para dar cuenta de la estructura de la razón se introduce un "como" que hace de tal figura una imagen que vale analógicamente y allí donde se apela a la razón para dar cuenta de los organismos vivientes se le otorga al juicio que de allí resulta validez solo reflexionante, en Hegel, tal doble direccionalidad adquiere una decidida radicalidad ya a partir de los escritos jenenses. De hecho, en Fe y saber (1802), el filósofo articula la idea kantiana de que la noción de Naturzweck constituye un principio de la razón que encuentra en los seres organizados de la naturaleza sus ejemplos en la experiencia con la idea de que el organismo no es otra cosa más que "razón real" (reelle Vernunft) (cfr. GW 04: 342; FyS: 83). Por otro lado, tal solidaridad plasma una estructura sistemática en la que espíritu y naturaleza no se contraponen, sino que el primero eleva a verdad la segunda, conteniéndola como momento constitutivo de su generarse. Será por otra parte este constituirse del espíritu en un comercio constante con la vida uno de los motivos que permita considerarlo como viviente.

Desde este punto de vista, la radicalización hegeliana de esa doble direccionalidad razón-organismo que encontramos en Kant resulta decisiva

\footnotetext{
${ }^{6}$ Estos términos resultan en general superpuestos en Hegel, mientras no parecería ser así en
} Kant; cfr. $A A$ 05: 374; CJ: 235. 
para la configuración del idealismo objetivo hegeliano ${ }^{7}$. En este sentido es suficiente con recordar que la concepción de la naturaleza como tal que tiene su ápice y su lugar de confluencia en la vida, como así también la consideración de la vida natural como realización sensible de la razón, resulta pendant imprescindible de la idea de la razón como "sustancia universal" que anima lo natural y lo espiritual y que exige un abandono de toda forma de dualismo. Así, escribe Frederick Beiser, en el enfoque hegeliano, la concepción organicista del universo resulta indispensable para poder superar el dualismo persistente entre sujeto y objeto propio del idealismo kantiano-fichteano cuya separación entre trascendental y empírico retomaría la oposición cartesiana entre res cogitans y res extensa. En este sentido, el concepto de organismo y la estructura finalista que el mismo supone sería decisivo para pensar el pasaje de la necesidad a la libertad y por lo tanto para pensar la posibilidad del realizarse de la razón, y de la exigencia de libertad que la constituye, en el mundo (Beiser 2005: 104). ${ }^{8}$ Es importante subrayar, en este contexto, que la asunción del modelo orgánico como paradigma comporta la concepción no solo de la naturaleza sino también de la razón en términos organicistas. Lo que intentamos decir es que la superación del dualismo kantiano se le presenta a Hegel como posible solo a partir, por un lado, del abandono de la concepción de la razón como facultad de un sujeto determinado constituido como ente racional finito y sin ninguna garantía de isomorfismo respecto del mundo, y, por el otro, de la contemporánea consideración de la razón como estructura inherente tanto a la subjetividad como a la objetividad. Estructura que ha de ser pensada como "sustancia universal" tanto de las cosas como de lo espiritual, como "Nous que gobierna el mundo" y que lo habita en el sentido organicista de proceso de realización de sí en el mundo y con él (cfr. Enc. \24).

Lo que hace posible este enfoque es la lectura de Natur y Vernunft a la luz del concepto de Zweck. La vida con su estructura finalística deviene entonces concepto clave para la comprensión de la razón y del mundo. Desde este punto de vista, la íntima solidaridad reconocida por Kant entre razón y vida o razón y organismo lleva a Hegel a considerar la vida como concepto metodológicamente decisivo para comprender la razón, ya no como simple facultad, sino como principio y esencia de lo real (cfr. GW 11:21; CdL, 47). De ahí la presencia y el rol clave de la vida en las tres partes del sistema

\footnotetext{
${ }^{7}$ Sobre el concepto hegeliano de idealismo objetivo, $c f r$. Halbig 2007: 33-60 y Halbig: 2002; Illetterati 2007: 1-20 e Illetterati 2016: 105-132; Soresi 2012; Ferrarin 2016.

${ }^{8}$ También Thomas Khurana habla del concepto de vida como "concepto crucial de transición” que apunta a garantizar el pasaje de la necesidad a la libertad (Khurana 2013: 19).
} 
maduro. De ahí la necesidad, por otro lado, de la vida como momento de la lógica aun cuando, como el mismo Hegel reconoce, pareciera tratarse de un objeto "tan real, que con ella puede parecer excedido el ámbito de la lógica” (GW 12: 178; CdL: 671). La pregunta que nos planteamos entonces es cuál es el significado y cuál el lugar de la vida en la Lógica, como así también qué implica tal ubicación de cara a la configuración del sistema y más específicamente, de cara a la relación entre naturaleza y espíritu.

\section{3.}

Ta cuestión de la aparición de la vida en el recorrido lógico puede responderse, en primera instancia, a partir de la consideración de la especificidad de la lógica hegeliana como "lógica especulativa", es decir, como tal que no se ocupa de determinaciones de pensamiento entendidas como formas indiferentes al contenido, estructuras rígidas y vacías, ajenas a la materia que solo la realidad puede proveer, la cual siendo pensada como originariamente independiente y heteromorfa respecto del pensar -situada "en la extremidad opuesta a la que nos hallamos nosotros y el pensamiento a ellas referido" (CdL: 35$)$-, resulta en última instancia inaccesible al mismo. En la medida en que "contiene el pensamiento, en cuanto este es también la cosa en sí misma, o bien contiene la cosa en sí, en cuanto esta es también el pensamiento puro" (GW 11: 20; CdL, 46), la lógica especulativa no puede abstraer de las formas reales, sino que es más bien la "red adamantina en la que transferimos todo material y solo así comenzamos a volverlo inteligible" (Enz. \$246Z, GW 20, trad. nuestra).

Así las cosas, la vida no podía no hallar su lugar en el desarrollo de las determinaciones puras del pensar. Mas la vida, en la lógica, no solo responde al presentarse de esta última como reconstrucción y organización de lo concreto en el ámbito del pensar (cfr. Illetterati 1995: 220-221); en la vida la idea tematiza su más íntima constitución. En la vida, la idea se aferra a sí misma en su inmediatez, se pone a sí misma en su estructura más inmediata, que no consiste en otra cosa más que en movimiento, devenir, actividad; más precisamente, automovimiento. Ahora bien, en tanto que movimiento que tiene su origen en sí mismo, la vida, escribe Hegel, es una "absoluta contradicción para la reflexión", es para ella "misterio incomprensible (unbegreifliches Geheimnis), porque la reflexión no comprende el concepto, y no lo concibe como sustancia de la vida" (GW 12: 181; CdL: 673). En cuanto contradicción, la vida es inaferrable para el entendimiento mas es, al mismo tiempo, aquello donde "comienza lo que es verdadero" (Enz. \$337Z, GW 20, trad. nuestra). Es por ello que la vida se presenta como el primer pasaje 
del último momento de la lógica que es "la idea". Esta última, como fundamento y sostén de todo el proceso precedente, accede a sí misma solo al final de tal proceso explicitando ahora su carácter más inmediato que es el de ser actividad y de actividad procesual; allí la idea accede finalmente a saberse como constituyéndose en tal actividad y con ella.

Tematizar su carácter de movimiento, devenir y actividad implica para Hegel, también, explicitar la contradictoriedad que habita y constituye la idea en tanto que vida o viviente. De hecho, a lo largo de toda la producción hegeliana, el concepto de vida aparece estrechamente vinculado al de contradicción y oposición. Decir que con la vida comienza la verdad significa decir que allí donde hay vida, hay también contradicción. La vida contiene la contradicción y se define como viviente aquella fuerza capaz de sostenerse en la contradicción. Tal superposición de vida y contradicción resulta no solo de la reapropiación hegeliana de la relación entre razón y antinomia en la primera Crítica -en virtud de la cual la razón ha de presentarse como la fuerza capaz de atravesar el Abgrund de la contradicción, no como facultad que sucumbe ante ella (cfr., GW 04:336; FyS: 78; y Enc. $\int S 48$ y 48Z)- sino también de una lectura de la tercera Crítica, en la que Kant se pregunta acerca de la contradictoriedad posible del concepto de fin natural, que él define como causa y efecto de sí ( $A A$ 05: 370; $C J$ : 230). Insistiendo en el "doble sentido" en el que ha de ser tomada esta reciprocidad causal implícita en el concepto de Naturzweck, Kant intenta evitar la contradicción, pues aquí el efecto es al mismo tiempo causa de sí como efecto en una línea causal que Kant llama ideal y no real (cfr. $A A$ 05: 371; CJ: 230). Hegel, en cambio, asume radicalmente el parentesco entre fin natural y causa sui, presentando esta última como contradicción y, justamente por ello, como concepto estrictamente racional. No como el lugar donde la razón se disuelve, sino como el lugar donde la razón comienza a comprenderse a sí misma.

Ya desde los escritos de Frankfurt, la contradicción se presenta como la peculiaridad de lo viviente. Así también en la Ciencia de la lógica, Hegel vuelve a identificar positividad con quietud y por lo tanto con lo muerto y negatividad con actividad y por ende con lo vivo:"En efecto, frente a ella [la contradicción], la identidad es solo la determinación de lo simple inmediato, del ser muerto; en cambio, la contradicción es la raíz de todo movimiento y vitalidad; pues solo al contener la contradicción en sí, una cosa se mueve, tiene impulso (Trieb) y actividad (Tätigkeit)" (GW 11: 286; CdL: 386). Criticando a todos aquellos que pretenden hacer de la contradicción algo que pertenece a la reflexión subjetiva y que es en el fondo impensable, Hegel afirma que "es lo negativo en su determinación esencial, el principio de todo automovimento, que no consiste en otra cosa sino en una manifestación de la misma contradicción" (GW 11:286; CdL: 386); solo allí donde hay nega- 
tividad, hay un sobresalir de sí, un modificarse, hay pues vida: "Por lo tanto algo es viviente, solo cuando contiene en sí la contradicción y justamente es esta fuerza de contener y sostener en sí la contradicción" (GW 11:287; CdL: 387). Y en una de las Vorlesungen der Logik se lee: "la quietud es la muerte: en la muerte ya no hay más contradicción. Toda necesidad, toda actividad comienza con un dolor o con una necesidad" (cit. en Sell 2013: 128; trad. nuestra). La contradicción es diferencia, oposición, falta, carencia y al mismo tiempo exigencia de superarla, de cerrar el agujero. De ahí que ella sea, para Hegel, la raíz del automovimiento, dando lugar, de esa suerte, a una ontología no del ser compacto y quieto sino de la actividad. ${ }^{9}$

La idea kantiana de la reciprocidad causal propia del Naturzweck abre a Hegel la posibilidad de una comprensión verdaderamente especulativa de la vida; sin embargo, como se sabe, la teleología kantiana resulta insuficiente, pues, en la medida en que Kant hace de tal saber un juicio solamente subjetivo o externo, nada garantiza acerca del ser efectivo de los seres organizados de la naturaleza. De ahí que para Hegel fuese necesario proceder más allá de Kant.Y para ello el filósofo de Stuttgart se sirve de la obra aristotélica. El estagirita no solo inaugura la posibilidad de entender la "naturaleza como vida, es decir, como algo que tiene un fin suyo y una unidad consigo misma", sino que además, ofreciendo un punto de apoyo teórico para pensar la actividad como "energeia" -como actuación de sí que es al mismo tiempo fin en sí mismo-, abre el camino para esa conjugación de razón y naturaleza - de pensar y real- que define el idealismo objetivo hegeliano. La estructura de la razón como Tätigkeit, que es Wirken -en el sentido de "efectividad activa (tätige Wirksamkeit), el escindirse a sí mismo, este ser para sí, superar la unidad y poner la escisión" ( $W$ 19: 155; Lecc.: 258; trad. ligeramente modif.)-, es la de una actividad que tiene su fin en sí, o, mejor aún, que es fin en sí y realización de aquel.Y como "fin en sí" tal energeia es, para Hegel, vida, pues "el profundo y verdadero concepto de lo vivo" es pensado como "un algo idéntico a sí mismo, que se repele a sí mismo y que, en su manifestación, permanece idéntico a su concepto"; es decir, concluye Hegel, como "la idea que se produce a sí misma" ( $W: 176$; Lecc.: 275).

La finalidad interna aristotélica, articulada con la noción kantiana de Naturzweck como causa sui, deviene entonces, en las manos de Hegel, actividad que se autodetermina, que se realiza en el sentido de in-formar de

\footnotetext{
9 “Todo viviente activo tiene en realidad la contradicción en sí, siendo sin embargo al mismo tiempo la resolución (Auflösung) de la contradicción. Yo soy lo afirmativo, y al mismo tiempo lo negativo, y tengo el sentir de ambos. Este sentir es la fuente de toda actividad, la satisfacción es la resolución de la contradicción" (GW 23/1: 231, cit. en Sell, 2013: 127).
} 
sí, autoconservándose y generando unidad consigo en todas sus relaciones; actividad de escindirse, diferenciarse y conservarse, de mantener su propia unicidad en ese movimiento y de producirse a sí mismo en él ( $c f r . W$ 19: 176; Lecc.: 275). Al mismo tiempo, en virtud de la torsión operada por Hegel sobre el concepto kantiano de fin, tal concepto deja de ser un concepto constitutivo de la razón, en el sentido de que la razón como facultad posee tal representación o contenido, para transformase en el concepto que define el ser mismo de la razón como tätige Wirksamkeit, como actividad que se realiza y que es solamente en este efectivo y concreto realizarse.

Si todo lo dicho anteriormente es cierto, la vida como idea en su existencia inmediata expresa la primera instancia en la cual la idea se aferra a sí misma como actividad, como movimiento de producción de sí que es al mismo tiempo movimiento de conservación de sí, en el medium de su escindirse, como contradicción y superación de la contradicción. Al mismo tiempo, en virtud de la articulación hegeliana de finalidad interna aristotélica y conceptos kantianos de Zweck y Naturzweck, la vida se presenta como concepto clave y decisivo de una concepción para la cual la razón no es otra respecto de la naturaleza sino que se encuentra en ella, pues comparte con ella una "parentesco estructural" (Sell 2013: 133). Ese doble movimiento que veíamos producirse en Kant, en virtud del cual se explicaba la razón a partir de la imagen del organismo y se explicaba el organismo recurriendo a un concepto constitutivo de la razón, es radicalizado en la perspectiva hegeliana: aquí la razón capta su propia estructura como fin en sí y, captándose de ese modo, se halla a sí misma en los vivientes naturales, que también son pensados sobre la base de aquella estructura. De aquí la modificación del estatuto de la lógica -que ahora es también metafisica-y la necesidad de la aparición en ella de la vida, identificada con la estructura lógica del Zweck. Al mismo tiempo, justamente porque la instancia en la que la relación finalística se muestra más inmediata y explícitamente es la naturaleza y sus formas vivientes, en la Lógica, como han puesto en evidencia varios intérpretes, el examen de la idea de la vida parece estar orientado a partir de los caracteres de la vida natural ( $c f r$. entre otros Düsing 1986: 280; Illetterati 1995: 232; Sell 2013: 118).

\section{4.}

Tno de los objetivos del presente trabajo es, como se dijo, leer, a partir del lugar de la vida en la lógica, el lugar y significado de la vida en el sistema. Sin necesidad de entrar en las discusiones acerca del estatuto de la lógica dentro del sistema, se podría decir que en Hegel la lógica se configura como exposición de un contenido en su hacerse forma 
de sí (cfr. Enc. 237). Ese contenido, al cual Hegel se refiere, sustantivándolo en el concepto de "lo lógico" (das Logische), es "impulso", "instinto" (Trieb) ( $f r$ r. GW 12: 238; CdL: 727) o fuerza que se afirma o realiza autocomprendiéndose como tal y tal proceso de autocomprensión es la lógica. En este sentido, para Hegel, das Logische es vida cuyo movimiento no consiste más que en el proceso de saberse. Esto no ha de ser entendido en el sentido de una supuesta autosuficiencia de la lógica, ni de su actor principal, la idea, como si naturaleza y espíritu no fueran sino una suerte de fenómeno, de aparición externa de un drama que se lleva a cabo en otro escenario. Como dice Remo Bodei, analizando los famosos tres silogismos con los que se cierra la Enciclopedia, el orden de sucesión de los parágrafos de la misma es solamente un orden didáctico y subalterno respecto al entero de la "razón que se piensa a sí misma"; lo cual no solo comporta que la progresión canónica del sistema enciclopédico -lógica, naturaleza, espíritu- no es vinculante, sino también que no hay realiter ningún pasaje entre la lógica y la naturaleza sino para la representación expositiva didáctica de la Enciclopedia, que no coincide con el sistema en cuanto tal (Bodei 2014: 352-361). Sobre todo, la lógica como razón que se piensa a sí misma, como pensar que se sabe, asume su valor solo como resultado de la experiencia de las ciencias ( $f$ r. GW 11:28; CdL 53) ${ }^{10}$ pues solo allí adquiere el significado de "universalidad verificada (bewaerht) en el contenido concreto como realidad efectiva suya" (Enc. \$574). Solo en ese retorno al propio inicio, en el que "lo lógico es así su resultado como lo espiritual", se alcanza la ciencia acabada y completa.

Por fuera de su conexión con las otras esferas en las que la idea se realiza, la lógica es entonces mirada unilateral y, en cierto sentido, incompleta. Incompleta, porque es expresión solo de una de las instancias o modos de realización de la idea; al mismo tiempo no se debe olvidar que, para Hegel, cada instancia o momento de la filosofía como ciencia es el todo del saber desde una perspectiva particular, "es la idea entera" (cfr. Enc. \247Z). Cada momento de la idea es, a su vez, la idea completa. En este sentido, la lógica, en tanto que "forma pura del concepto que intuye su propio contenido como sí misma" (Enc. \237), condensa y organiza tal contenido en el elemento del pensar puro, en la rarefacta transparencia de las formas inteligibles anilladas en un desarrollo necesario e inmanente, de suerte que aquí aparecen, en relación de continuidad, las categorías o esencias conceptuales que se despliegan en el mundo pero depuradas de tal realización.

\footnotetext{
${ }^{10}$ Hay que reconocer que muchas veces es el mismo Hegel el que alimenta la idea de una cierta autosuficiencia o por lo menos superioridad de la lógica sobre las otras formas de exposición de sí de la Idea ( $f r$ r. en este sentido GW 12: 237; CdL: 726).
} 
Si lo dicho es plausible, esto significa que la lógica es "reconstrucción" del movimiento total de la idea desde la perspectiva de las determinaciones puras del pensar. Hay, entonces, por un lado, una especificidad de la lógica; por el otro, las categorías lógicas, aun siendo primariamente expresión de una determinada estructura relacional desde la perspectiva del pensar puro, resumirían también los caracteres y conexiones de tal estructura en el ámbito natural y espiritual, es decir, mostrarían, en el tejido de las determinaciones puras del pensar, una estructura que habrá de encarnarse en objetos y formas del mundo. En virtud de la especificidad señalada, Hegel insiste sobre las diferencias entre perspectiva lógica y la perspectiva científica de la vida, tanto de la vida en conexión con el espíritu como de esta en cuanto vida biológica o natural ( $c f r$. GW 12: 180; CdL: 672). En virtud, en cambio, de la condensación lógica de formas naturales y espirituales, podemos decir que el lugar que cada categoría ocupe en la necesaria evolución lógica "corresponde" al lugar de tal estructura relacional en el todo sistemático de la razón que se sabe a sí misma. Es decir, el lugar de la idea lógica de la vida puede ser leído como perspectiva desde la cual comprender el lugar de la vida dentro del todo sistemático y por ende como ángulo a partir del cual comprender la relación entre vida y pensar. Para ello es entonces necesario determinar también qué está en juego en el pasaje de la idea como vida a la idea absoluta.

Como se vio, la vida es idea en su existencia inmediata, que a través de los tres momentos de la individualidad viviente, del proceso vital y del género, accede a constituirse como idea que se refiere a sí misma como idea, es decir, se desarrolla en la "idea del conocer". Que este pasaje resuma, en el ámbito lógico, el movimiento sistemático de la naturaleza al espíritu, lo da a entender el mismo Hegel abriendo el capítulo sobre "la idea del conocer" con distintos párrafos dedicados a las diferentes ciencias del espíritu, como antropología, fenomenología y psicología, las tres ciencias del espíritu subjetivo en la Enciclopedia (GW 12: 196-199; CdL: 687-689). No solo; Hegel escribe aquí explícitamente, no sin cierta incomodidad, que "en la conexión de esta exposición lógica, la idea de la vida es aquella de la cual ha surgido la idea del espíritu, o, lo que es lo mismo, aquella de la que ha sido probado que la idea del espíritu constituye la verdad" (GW 12: 196; CdL: 687). ${ }^{11}$ Allí donde la

${ }^{11}$ Se intensifica en estas páginas la tensión entre esfera lógica y esfera de las así llamadas ciencias reales: por un lado, los dos niveles aparecen constantemente superpuestos, por el otro, Hegel insiste acerca de la diferencia entre ellos, acentuando una separación que corre el riesgo de llevar hacia una concepción de la lógica como autosuficiente: "Dado que representa tal resultado, esta idea [la idea del espíritu que surge de la idea de la vida] tiene en sí y por 
idea elimina la individualidad abstracta y es idéntica consigo misma, es decir es, como género, igual a sí misma, la idea es el espíritu. La idea del conocer es entonces la idea como espíritu, aun cuando Hegel insista en que aquí el espíritu es considerado solo en la forma que compete a esta idea como forma lógica, no en sus concreciones empíricas (GW 12: 196; $C d L$ : 687).

Ahora bien, lo que impone el pasaje de la vida al conocer es la irrealizabilidad, en el contexto de la vida, de una adecuada articulación de universalidad y particularidad, de suerte que la vida queda atrapada en el círculo de un eterno retorno de lo idéntico, sin historia ni progreso ${ }^{12}$ : “[ ...] El viviente inmediato se media en el proceso del género consigo mismo y se eleva así por encima de su inmediatez, pero solamente para volver a recaer en ella. Así las cosas, la vida se despliega en principio solamente en el mal infinito de un progreso al infinito" (Enc. $\$ 221 Z$ trad. nuestra, curs. nuestro). A través de la reproducción, el viviente singular intenta acceder a la universalidad del género, pero no hace sino reproducirse en un nuevo viviente singular, destinado a repetir el ciclo desde el inicio.Y la única instancia en la que el individuo supera su existencia inmediata e indiferente es entonces su perecer en la unidad negativa del género: "El viviente muere porque es la contradicción de ser en sí lo universal, el género, existiendo sin embargo inmediatamente solo como singular. En la muerte el género demuestra ser la potencia sobre el individuo inmediato [...] Pero el animal no accede a ser para sí en su género, sino que sucumbe a la potencia del género" (Enc. $\$ 221 \mathrm{Z}$ trad. nuestra; cfr. también GW 12: 191; CdL: 682). Al mismo tiempo, este perecer de las particularidades separadas propias de la vida individual en el género es la superación de la individualidad y el fusionarse del género consigo mismo y con ello el hacerse por sí misma de la universalidad de la idea con lo cual esta deviene idea del conocer ( $c f r . G W 12: 191$; CdL: 681; Enc. $\$ \$ 221 \mathrm{Z}$ y 22).

La idea del conocer, escribe Hegel, es el juicio de la idea, la escisión de esa inmediatez que es la vida. Como tal, la idea del conocer se desdobla en idea teórica e idea práctica para recogerse nuevamente en la idea que se piensa a sí misma, es decir la idea absoluta, como "unidad de la idea de la vida y de la idea del conocer" (Enz. $\$ 236 Z, G W 20$, trad. nuestra). La idea

sí misma su verdad, con la que puede después compararse también el aspecto empírico, o sea el fenómeno del espíritu (Erscheinung des Geistes), para ver cómo coincide con ella. Sin embargo, el elemento mismo puede comprenderse solo por medio de la idea y sobre la base de esta" (GW 12: 196; CdL: 687).

12 “[...] y entre el mundo natural y el espiritual subsiste además la diferencia que, mientras el mundo natural solo retorna continuamente a sí mismo, en el mundo espiritual se da un progreso" (Enz. \234Z, GW 20, trad. nuestra). 
absoluta es "forma pura" en el sentido de que intuye su propio contenido como ella misma o que es contenido para sí.Y de esa suerte, escribe Hegel, la idea absoluta es fin (Zweck), pero como tal que recoge en sí la totalidad del proceso, de todo el movimiento que la ha llevado hasta allí -"la totalidad del decursus vitae" (Enc. \$237Z)-. Desde este punto de vista, la idea absoluta es un "retorno a la vida", pero un retorno que ha superado la forma de su inmediatez y que por lo tanto es "libre concepto subjetivo, existente por sí, y que, por ende, tiene la personalidad" (GW 12: 236; CdL: 725).

La perspectiva lógica confirma entonces que la vida, aun resultando la primera, inmediata forma de existencia de la idea, no es su forma más alta y verdadera. Con la vida comienza lo verdadero, pero la verdad, como escribía Hegel en el Prólogo de la Fenomenología, no es moneda fija, lista para su uso e intercambiabilidad, sino que es un movimiento que se despliega en una jerarquía de formas cada vez más perfectas. En el progreso de esas formas, la vida representa el eslabón más alto: en tanto que actividad, movimiento de autoconservación de sí en lo otro de sí, la vida es lugar de emergencia de la idea, mas no fundamento, ni fin último.Y así como la vida lógica no es el momento más alto de la idea en el recorrido de las determinaciones puras del pensar, la vida biológica o natural no es el momento más alto en la estructuración sistemática. El espíritu se configura como la verdad de la naturaleza y por lo tanto de la vida, en la medida en que esta última aparece como el vértice al que la naturaleza accede. Al mismo tiempo, en tanto que verdad de la naturaleza, el espíritu la contiene, superándola y edificando su propia realidad a partir y desde la naturaleza y no contraponiéndose o enajenándose de ella.

Desde este punto de vista, el espíritu no es algo otro de la vida o de la naturaleza, sino que se presenta como una "segunda naturaleza" (cfr. Enc. $\$ 410$ ), aun cuando esto no comporte ni identificación, ni reducción del espíritu a la naturaleza, como así tampoco una simple continuidad de grado entre espíritu y naturaleza, como si el primero fuese una complejización organizativa de la segunda. Revela el movimiento conceptual que Hegel tiene en mente el agregado al parágrafo 379 de la Enciclopedia:

El germen de la planta - este concepto presente en forma sensible- concluye su propio desplegarse con una realidad efectiva igual a él: la producción de la semilla. Lo mismo vale para el espíritu: también su desarrollo ha alcanzado su propia meta cuando el concepto del espíritu accede a su completa realización, o -lo que es lo mismo- cuando el espíritu accede a la plena conciencia de su propio concepto.

Esta identidad entre viviente y espíritu, basada en el hecho de que ambos consisten en una efectivización o realización de sí, de lo que cada 
uno es en su propio germen, se rompe, sin embargo, porque solo en el espíritu, según Hegel, autoproducción y autolimitación se realizan de manera acabada y completa:

Este coincidir del inicio y del fin (Dies sich-in-Eins-Zusammenziehen des Anfang mit dem Ende), este volver a sí mismo del concepto en su propia efectiva realización, en el espíritu se presenta de manera más completa que en el simple viviente; de hecho, mientras en el viviente el germen producido no se identifica con aquello de lo cual deriva, en el caso del espíritu que se conoce a sí mismo, lo producido es una unidad con lo que produce (Enz., \ 379Z, GW 20, trad. nuestra).

Si en la primera cita se insiste en que la planta, como todo ser viviente, es concepto en forma sensible, es presencia sensible del concepto, y se compara el desarrollo de la planta con el desarrollo del espíritu, el segundo pasaje, en cambio, pone una diferencia jerárquica entre organismo y espíritu, porque solo el espíritu, en tanto que accede al pleno saber de sí, es concepto adecuado a sí mismo o concepto realizado acabadamente; instancia inaccesible para el organismo animal, porque, como ya sabemos, en el viviente natural, la reproducción no comporta una adecuación de la individualidad a la universalidad del género, sino una recaída en una nueva individualidad que abre así a una circularidad estática, a un eterno retorno de lo idéntico que es propio de la naturaleza. Ahora bien, después de haber puesto en evidencia la superioridad del espíritu sobre la naturaleza, Hegel parece volver a mezclar las cartas, estableciendo que justamente en tanto que coincidencia acabada de inicio y fin, el espíritu es lo verdaderamente viviente:

El desarrollo completo del espíritu no es otra cosa más que su elevarse desde sí mismo a su propia verdad, y las así llamadas facultades del espíritu no tienen otro sentido que el de constituir los escalones de esta elevación. Mediante esta autodiferenciación, autotransformación, y mediante la conducción de las propias diferencias a la unidad del propio concepto, el espíritu es verdadero y viviente, orgánico y sistemático, y solo mediante el conocimiento de su naturaleza la ciencia del espíritu es también ella verdadera y viviente, orgánica y sistemática (Enz., \$379Z, GW20, trad. nuestra).

Pongamos ahora el foco sobre este coup de théâtre del discurso hegeliano. En primer lugar, puede ser interesante recordar que de este desdoblamiento de la vida en relación al espíritu ya daban cuenta las páginas del capítulo lógico sobre la vida. Allí Hegel, insistiendo sobre las diferencias entre perspectiva lógica y perspectiva científica de la vida, y después de haber 
afirmado que en la naturaleza la vida aparece arrojada a la exterioridad del subsistir y tiene como presupuesto la naturaleza inorgánica mientras que la idea lógica de la vida tiene como presupuesto el concepto, afirma que en el caso del espíritu, el mismo mantiene una doble relación con la vida, la cual es por un lado medio para el espíritu que la contrapone a sí y por el otro es en unidad con el espíritu que es "individuo viviente" (GW 12: 180; CdL: 672). Se trata entonces de entender el significado de esta doble relación.

La primera parte de la cita enciclopédica refleja, sin dudas, en el contexto sistemático, la ubicación de la vida en el recorrido lógico: la caracterización lógica de la vida como existencia inmediata de la idea y como primer momento de la última instancia del recorrido lógico comporta, en el plano de las ciencias, la no exclusión de la naturaleza -y sobre todo de la vida como momento más alto de la misma- del movimiento propio del Absoluto y por lo tanto de la verdad. Al mismo tiempo, la incompletitud lógica de la idea de la vida, en virtud de la cual la misma necesitaba mediarse a sí misma a través del conocer teórico y práctico, resultando así realización insuficiente de la idea, anticipaba la definición del espíritu como verdad de la naturaleza y, desde este punto de vista, como instancia de "superación" de la misma. El espíritu supera la vida pues mientras que esta última queda atrapada en una circularidad horizontal y estática en la que no hay verdadera conjugación de universal y particular, el primero progresa hacia tal conjugación, de suerte que solo aquí se accede finalmente a la persona y por ende a la libertad.

En segundo lugar, justamente porque el espíritu se presenta como un volver a sí desde la naturaleza y como un mostrarse como la verdad de la misma, al mismo tiempo que se reconoce a la naturaleza una estructura finalista afin a aquella sobre la que se constituye el espíritu, es posible superar una concepción del espíritu como ajeno o contrapuesto a la naturaleza; es decir, es posible superar el dualismo naturaleza-espíritu, según Hegel vigente en la tradición trascendental de Kant a Fichte. El espíritu hegeliano no se define entonces por un "sacarse de encima" la naturaleza, ni puede constituirse por fuera de ella o independientemente de ella. El espíritu se constituye como tal solo en un constante hacer las cuentas con la naturaleza, solo en tanto que retorno a sí desde la vida natural, que no es sin su contemporáneo "retorno a la vida" (GW 12: 236; CdL: 725) que según Hegel define la idea absoluta. Desde este punto de vista, el primer significado que las palabras de la última cita del agregado al \$379 arrojan es que el espíritu es viviente y orgánico justamente en tanto que se constituye solo en este constante comercio con su otro -la naturaleza y por lo tanto la vida natural-, en la irresoluble y al mismo tiempo soportada tensión dialéctica entre exterioridad y interioridad, entre necesidad y carencia y entre apropiación y asimilación. 
El espíritu, aun en sus formas más altas, es siempre un constante retorno al otro -la naturaleza, la vida- y desde el otro.

En nuestra lectura el espíritu es viviente y orgánico, entonces, en dos sentidos diferentes: en primer lugar, porque no rechaza la vida natural, sino que esta es momento constitutivo suyo. El espíritu se hace tal a partir de la vida biológica, recogiéndola en sí, pero diferenciándose de ella, superándola, mas en un constante comercio con ella. En segundo lugar, el espíritu es viviente justamente en virtud de su constituirse mediándose con su propio otro, pues apropiación y asimilación de lo otro de sí, conjuntamente con autogeneración, autodiferenciación, autoorganización y reciprocidad parte-todo son caracteres definitorios de lo orgánico y viviente. El espíritu es procesualidad circular que vuelve sobre sí desde lo otro de sí. Tal movimiento se presenta como curvarse sobre sí misma de la causa, como reciprocidad de causa y efecto y siendo esto lo que define el concepto de Zweck, espíritu y vida parecen encontrar en él su lugar de confluencia. En este segundo sentido, el espíritu es organismo y viviente de una manera diferente a como lo es el viviente natural, pues, como vimos, mientras la vida natural comporta una "recaída" en un idéntico que es otro numéricamente distinto, el espíritu es fin completamente realizado, en el que meta e inicio coinciden acabadamente.

\section{5.}

Como reflexión conclusiva del recorrido precedente podemos decir lo siguiente entonces: el concepto de organismo, como bien sostienen Karen Ng y Annette Sell, no tiene un carácter simplemente metafórico o analógico en Hegel. Los conceptos de organismo y de vida -que aparecen en general superpuestos en el discurso hegeliano- cumplen una función metodológica y sistemática decisiva en el enfoque del filósofo de Stuttgart, permitiendo una articulación de la relación razón-mundo y espíritu-naturaleza que apunta a superar toda forma de dualismo. En Hegel, como se vio, la reciprocidad del movimiento kantiano entre razón y vida se radicaliza, articulándose en un doble sentido: por un lado, la vida orgánica y natural se presenta como efectiva realización de la razón, como "razón en forma sensible" (Enc. \$379Z) o "razón real" (GW 04: 342; FyS: 83), de suerte que, no habiendo alteridad entre ellos, es posible acceder a una inteligibilidad completa y determinante del viviente. En los individuos vivientes la razón se halla efectivamente a sí misma, ya no, como en Kant, como facultad subjetiva, sino como fuerza que se despliega objetivamente. Por otro lado, justamente en tanto que fuerza que se despliega y en ese desplegarse 
se autoconstituye, la razón es proceso de realización de sí. Por ello, ha de ser pensada como organismo viviente, pues, como aquel, no se presenta como una identidad estática sino en itinere, una identidad que se mantiene como tal aun en el proceso de autodiferenciación y enajenación de sí o comercio con lo otro de sí. Aquí movimiento no es disipación o dispersión de la unidad inicial sino que en tal despliegue la razón se mantiene siempre en unidad consigo y trabaja para ella misma. La razón hegeliana es proceso, en el que hay un mantenerse de la identidad inicial y es identidad concreta porque esta identidad se construye en el movimiento de su propio desplegarse.

Si la razón es organismo, también lo será el espíritu en tanto que instancia más acabada de realización de la razón. De ahí que, desde el punto de vista sistemático, la radicalización del movimiento de doble direccionalidad de razón-vida implique la consideración del espíritu como viviente. En el recorrido precedente, sin embargo, se intentó mostrar que el espíritu es viviente en dos sentidos diferentes que aparecían ya condensados en la doble ubicación de la vida en la esfera lógica. El espíritu es viviente porque es la verdad de la naturaleza, que tiene su génesis en ella y no se produce sin ella, siendo al mismo tiempo un constante retornar en la naturaleza y desde ella. Es decir, es viviente en el sentido de que "recoge" en sí la vida -como instancia mas alta de la naturaleza- volviéndola para sí.Y el espíritu es viviente en el sentido de que encarna la razón como proceso-movimiento, actividad-de realización de sí mediante lo otro de sí. En el primer sentido, como se decía en la lógica, la vida es un medio constitutivo del espíritu. En el segundo, el espíritu es viviente porque ahora la vida es, como también decía la lógica, toda una con él.

Cabe señalar, sin embargo, un punto de tensión en el cuadro recién delineado. En tanto que fin en sí, en tanto que movimiento circular de retorno a sí desde lo otro de sí, el espíritu es, se desarrolla y puede ser pensado como orgánico y como viviente. Al mismo tiempo, la verdad en tanto que proceso de realización de sí, no se realiza, en Hegel, en el espacio circular de un movimiento puramente orgánico sin dirección ni progreso, pues implica perfeccionamiento de sus formas y proyectualidad (Enc. \$234Z). Es ahí donde la circularidad estática del Zweck -o más precisamente del Naturzweck-, con su inmanente reciprocidad de causa y efecto, de todo y parte, no puede agotar la realidad propia del espíritu, que no es solo fin en sí, sino también para sí. Ese para sí introduce un desnivel, un "contrapunto" en las palabras de Wladimiro Giacché (1990: 14), entre finalidad y subjetividad. El espíritu es organismo en tanto que Zweck, pero no es solo organismo. Es procesualidad circular, pero no es una circularidad congelada en un eterno retorno de lo mismo. El espíritu es la instancia capaz de "agujerear" la circularidad horizontal y por ende repetitiva del fin natural. La procesualidad orgánica, en este sentido, no agota la realidad del espíritu; y no lo hace pues 
en el espíritu los términos en relación no se limitan simplemente a esa reciprocidad causal que los confinaría a una intercambiabilidad indiferente sin dirección ni movimiento escalar o "hacia delante".

Desde este punto de vista, nos parece que el movimiento circular, en el cual el fin se recurva sobre el inicio, que caracteriza la estructura del organismo, convive en Hegel con la exigencia de un "progreso" hacia delante, con la necesidad de un poner fines y actuar en conformidad con ellos. Y si el espíritu, como se dijo, es instancia más acabada de expresión de la razón, también en esta encontraríamos el mismo desnivel en virtud del cual la razón parece presentarse como organismo y, al mismo tiempo, parece no dejarse agotar por la imagen de lo orgánico. En la Vorrede de la Fenomenología, de hecho, la razón es presentada ya sea recurriendo a la figura de la planta que es todo lo que está contenido en el germen de la misma, ya sea como un obrar en conformidad a fines que no necesariamente les son dados $a b$ initio. Tal tensión ya ha sido evidenciada por Alfredo Ferrarin, quien subraya la existencia de dos modelos antagonistas de razón en Hegel: el "modelo orgánico" y el "modelo poiético o arquitectónico”: “en el modelo orgánico -dice el estudioso italiano- el cuerpo viviente tiene ciertamente una finalidad interna, mas la misma le es dada, y cada miembro de una especie lleva a cabo naturalmente su curso; en el modelo arquitectónico, en cambio, se presupone una individualidad que se distingue de la especie para producir algo nuevo" (Ferrarin 2016: 33).

Así expuesta -como tensión entre razón presentada como fuerza autónoma que opera a través del yo y que no puede no realizar lo que ella misma es en germen, y una razón como arquitecto que pone conscientemente fines resolviéndose en la subjetividad de un yo individual- la cuestión parece remitir, en parte, al menos, al tema de la apertura o del cierre del sistema; temática que obviamente excede los límites del presente trabajo. Es evidente, sin embargo, que la imagen del organismo parece presentar la verdad sí como proceso, mas como proceso cerrado y dominado por una necesidad preestablecida y a esta idea de un proceso congelado y ensimismado en su propia circularidad parece contribuir también la imagen hegeliana de la idea absoluta como "vida imperecedera" (unvergängliches Leben) (GW 12: 236; CdL: 725). La imagen del arquitecto parecería garantizar en cambio la apertura de un espacio de novedad, necesario para que el proceso no se congele en el movimiento puramente espacial de la circularidad.

Mientras que la figura del fin-organismo, presentando la razón como desarrollo de lo que ya es ab initio, expresa la idea de la misma como fuerza que se despliega objetivamente, la imagen de la razón que pone fines parecería dar cuenta del sujeto que en tanto que racional apunta en su obrar a realizar la razón históricamente. En este caso se pone en evidencia que 
la razón no es sino en su efectivo encarnarse en subjetividades espirituales, individuos dotados de razón que obran en pos de un hacer de la razón la sustancia del mundo. Sin pretender desdibujar o anular la tensión señalada, quizás se pueda sugerir que todo el esfuerzo de Hegel reside en un habitarla, sosteniendo que la razón misma es un "tener juntas", como "antagonistas-colaboradoras" (Bodei 2014), esas dos diferentes estructuras relacionales. Y es justamente desde esa tensión que la filosofia hegeliana sigue ofreciéndonos instrumentos para leer nuestro presente y los terribles desafios que presenta.

\section{BIBLIOGRAFÍA}

Achella, S. (2020), Pensare la vita. Saggio su Hegel (Bologna: Il Mulino).

Beiser, F. (2003), "Hegel and Naturphilosophie", Studies in History and Philosophy of Science, 32: 135-147.

Beiser, F. (2005), Hegel (New York: Routledge).

Bodei, R. (2014), La civetta e la talpa: Sistema ed epoca in Hegel (Bologna: Il Mulino).

Chiereghin, F. (1990), "Finalità e idea della vita; La ricezione hegeliana della teleologia in Kant", Verifiche, 19: 127-229.

Düsing, K. (1986) “Die Idee des Lebens in Hegels Logik”, en R. P. Horstmann y J. M. Petry (1986) (eds.), Hegels Philosophie der Natur: Beziehungen zwischen empirischer und spekulativer Naturerkenntnis (Stuttgart: Klett Cotta, 276-289).

Edmundts, D. y Horstmann, R.-P. (2002), G.W.F. Hegel: Eine Einführung (Stuttgart: Reclam).

Ferrarin, A. (2016), Il pensare e l'io: Hegel e la critica di Kant (Roma: Carocci).

Fonnesu L. y Ziglioli L. (2016) (eds.), System und Logik bei Hegel (Berlin: Georg Olms).

Giacché, W. (1990), Finalità e soggettività: Forme del Finalismo nella Scienza della logica di Hegel (Genova: Pantograf).

Hahn, S. S. (2007), Contradiction in Motion: Hegel's Organic Concept of Life and Value (Ithaca - Londres: Cornell University Press).

Halbig, Ch. (2002), Objektives Denken: Erkenntnistheorie und Philosophy of Mind in Hegels System (Stuttgart - Bad Cannstatt: Frommann).

Halbig, Ch. (2007), "Pensieri oggettivi”, Verifiche. L'oggettività del pensiero. La filosofía di Hegel tra idealismo, anti-idealismo e realismo, 36 (1-4): 33-60.

Hegel, G. W. F. (CdL), Ciencia de la lógica, trad. de R. Mondolfo (Buenos Aires: Solar-Hachette, 1976) (cit. como $C d L$ seguido por número de página).

Hegel, G. W. F. (EJ), Escritos de juventud, trad. de J. M. Ripalda (México: FCE, 1978) (cit. como EJ seguido por número de página).

Hegel, G. W. F. (Enc.), Enciclopedia de las ciencias filosóficas en compendio, trad. de A.Valls Plana (Madrid:Alianza, 2005) (cit. como Enc. seguido por número de parágrafo). 
Hegel, G. W. F. (FdE), Fenomenología del espíritu, trad. de A. Gómez Ramos (Madrid: Abada, 2010) (cit. como FdE seguido por número de página).

Hegel, G. W. F. $(F y S)$, Fe y saber, trad. de V. Serrano (Madrid: Biblioteca Nueva, 2007) (cit. como $F y S$ seguido por número de página).

Hegel, G. W. F. (GW) Gesammelte Werke, in Verbindung mit der Hegel-Kommission der Rheinisch-Westfälischen Akademie der Wissenschaften und dem Hegel-Archiv der Ruhr-Universität Bochum (Hamburg: Meiner, 1968 ss.).

Hegel, G. W. F. (1979) Werke in 20. Bänden, E. Moldenhauer-K.M. Michel (ed.) (Frankfurt: Suhrkamp).

Hoffmann, T. (2014), Hegel: una propedéutica, trad. de M. Maureira y K. Wrehde (Buenos Aires: Biblos).

Horstmann, R.-P. y Petry, J. M. (1986) (eds.), Hegels Philosophie der Natur: Beziehungen zwischen empirischer und spekulativer Naturerkenntnis (Stuttgart: Klett Cotta).

Illetterati, L. (1995), Natura e ragione: Sullo sviluppo dell'idea di natura in Hegel (Trento: Pubblicazioni di Verifiche).

Illetterati, L. (2007), "L'oggettività del pensiero. La filosofia di Hegel tra idealismo, anti-idealismo e realismo: Un'introduzione", Verifiche. L'oggettività del pensiero. La filosofía di Hegel tra idealismo, anti-idealismo e realismo, 36, (1-4): 13-32.

Illetterati, L. (2016), “The Thought of Logic”, en L. Fonnesu y L. Ziglioli (2016) (eds.), System und Logik bei Hegel (Berlin: Georg Olms, 105-129).

Kant, I. $(A A)$, Gesammelte Schriften (Berlin: Walter de Gruyter, 1900 ss.) (cit. como $A A$ seguido del número de vol. y del número de pág.; para la Crítica de la razón pura se usan las siglas tradicionales de $K r V$ A y B para la segunda y segunda edición respectivamente, seguidos por el número de pág. de la edición original).

Kant, I. (CJ), Crítica de la facultad de juzgar, trad. de P. Oyarzún, (Caracas: Monte ÁvilaLatinoamericana, 1992) (cit. como CJ, seguida por número de página).

Kant, I. (CRP) Crítica de la razón pura, estudio preliminar, traducción y notas de M. Caimi (Buenos Aires: Colihue, 2010) (cit. como CRP, seguida por número de página).

Khurana, T. (2013) (ed.), The Freedom of Life (Berlin: August Verlag).

Kreines, J. (2013), "Kant and Hegel on Teleology and Life from the Perspective of Debates about Free Will”, en T. Khurana (2013) (ed.), The Freedom of Life (Berlin: August Verlag, 111-154).

Ng, K. (2020), Hegel's Concept of Life: Self-Consciousness, Freedom, Logic (Oxford: Oxford University Press).

Palermo, S.V. (2017), "Conformità a scopo, contingenza e intelletto intuente. La critica di Hegel alla lettura kantiana di Spinoza in Glauben und Wissen”, Rivista di Storia della filosofia, 72, nuova serie, (2): 245-265.

Pippin, R. (1989), Hegel's Idealism: The Satisfaction of Self-Consciousness (Cambridge: Cambridge University Press).

Sell, A. (2013), Der lebendige Begriff: Leben und Logik bei G.W.F. Hegel (Freiburg - Munich: Karl Alber). 
Soresi, S. (2012), Il soggetto del pensiero: Modi e articolazioni della nozione di pensiero in Hegel (Trento: Pubblicazioni diVerifiche).

Recibido: 29-04-2020; aceptado: 08-09-2020 